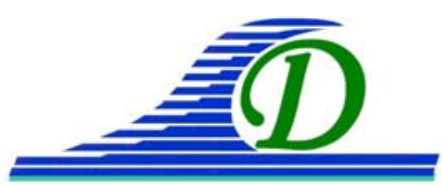
XIII ${ }^{\text {èmes }}$ Journées Nationales Génie Côtier - Génie Civil
Dunkerque, 2-4 juillet 2014

DOI:10.5150/jngcgc.2014.032 @ Editions Paralia CFL

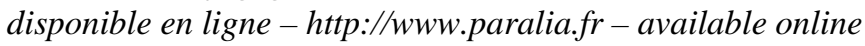

\title{
Modélisation de l'évolution morphodynamique des dunes sous-marines
}

\author{
Arnaud DORE ${ }^{1}$, Philippe BONNETON ${ }^{1}$, Vincent MARIEU ${ }^{1}$, Thierry GARLAN $^{2}$ \\ 1. Université de Bordeaux; CNRS; UMR 5805-EPOC, Talence, F-33405, France. \\ 2. SHOM ; HOM/REC-CFuD/Sédimentologie, CS 92803, 29228 BREST Cedex 2, \\ France. \\ a.dore@epoc.u-bordeaux1.fr
}

\section{Résumé :}

L'évolution morphodynamique des dunes hydrauliques (en courant stationnaire) est simulée à l'aide d'un modèle numérique bidimensionnel non-hydrostatique. La résolution de façon rétroactive de l'hydrodynamique, des flux sédimentaires et de la morphodynamique permet d'intégrer la phénoménologie non linéaire d'évolution du profil de fond. Les scénarios des modélisations sont tirés d'expérimentations en laboratoire reproduisant l'évolution du lit de sable faiblement perturbé soumis à un courant stationnaire jusqu'à la saturation du champ de dunes en équilibre avec l'écoulement. Les simulations réalisées permettent de modéliser les processus non linéaires d'auto organisation du fond et d'expliquer dans le détail le mécanisme clé d'appariement entre les formes sableuses. Les phases d'évolution sont reproduites avec tout d'abord l'apparition des premières rides d'instabilité, suivie d'une phase d'appariements en cascade, aboutissant à un champ de dunes en équilibre dynamique avec l'écoulement. Les ordres de grandeurs obtenus sont en accord avec les données expérimentales.

Mots-clés : Dune, Ride, Flux, Sédiments, Cisaillement, Turbulence, Appariement, Saturation, Auto-organisation, Modélisation.

\section{Introduction}

Un lit plan de sable soumis à un écoulement stationnaire est instable si localement la force de cisaillement dépasse le seuil de mise en mouvement du sédiment à un instant donné ( $\theta>\theta_{c}$ en un point du domaine). L’évolution du fond sableux issue de la perturbation initiale suit plusieurs étapes, exhibant en premier lieu des structures primitives de l'ordre de quelques grains de sable, puis donnant naissance à un premier champ de rides asymétriques développant un front d'avalanche, s'auto organisant à leur tour en formes sableuses de taille de plus en plus importante (longueur d'onde $\lambda$ et hauteur $H$ ) jusqu'à obtenir un champ de dunes en équilibre avec l'écoulement.

L'instabilité d'un lit de sable érodable soumis à un courant stationnaire a fait l'objet de recherches depuis près d'un demi-siècle, notamment à travers l'analyse de la stabilité linéaire des équations aux petites perturbations. Ces études montrent notamment que 


\section{Thème 2 - Dynamique sédimentaire}

l'instabilité résulte d'un déphasage entre le transport sédimentaire saturé et le courant moyen (KENNEDY, 1963), conséquence de l'effet de la friction de l'écoulement au fond (ENGELUND, 1970). Pour des formes de fond de longueurs d'ondes importantes (k.H O(1)) FOURRIERE et al. (2010) démontrent que les modes sont stables et établit la conclusion que les dunes ne sont pas générées par instabilité linéaire et se distinguent ainsi des rides.

Les expériences en laboratoire (COLEMAN \& MELVILLE, 1994 ; COLEMAN et al., 2003 ) ou en nature (FOURRIERE et al., 2010) montrent que la génération d'un champ de dunes suit une phénoménologie fortement non linéaire, les formes sableuses évoluant par appariements successifs pour donner naissance à des structures de plus grandes longueurs d'ondes. L'évolution en hauteur est plutôt reliée à une dune individuelle alors que le changement de longueur d'onde est dû à l'interaction des structures entre elles (NIEMANN et al., 2011). La modélisation numérique permet d'intégrer cette phénoménologie non linéaire. Cependant, il existe peu d'études de modélisation de l'évolution morphodynamique des dunes, et les approches utilisées n'ont pas permis de reproduire l'évolution du fond faiblement perturbé jusqu'à l'équilibre. (GIRI \& SHIMIZU, 2006) parviennent à simuler l'évolution à partir d'un fond faiblement perturbé mais les simulations ne montrent pas l'état de saturation. (NIEMANN et al., 2011) utilisent un modèle non-hydrostatique et un modèle de fermeture de la turbulence. Un filtre numérique est utilisé pour amortir les instabilités du modèle, ce qui redistribue artificiellement le sédiment, et les instabilités semblent demeurer. La modélisation d'un scénario tiré d'une expérimentation en laboratoire n’atteint pas la saturation et ne modélise pas le fond faiblement perturbé.

Dans ce papier, nous présentons un modèle capable de simuler l'évolution morphodynamique du champ de formes sableuses à partir d'un fond faiblement perturbé jusqu'à la saturation en reproduisant les mécanismes physiques observés lors des expérimentations en laboratoire.

\section{Modèle numérique}

Le code de calcul utilisé est une version évoluée de DUNE2D, développé initialement par (TJERRY, 1995) au sein de l'université technique du Danemark (DTU). Le modèle est composé de trois modules : le module hydrodynamique qui résout les équations de Navier Stokes dans la couche limite ainsi que la turbulence via une fermeture à deux équations, le modèle de transport sédimentaire qui calcule le transport par charriage et en suspension, et le module morphologique qui résout l'équation d'évolution du fond.

\subsection{Module hydrodynamique}

L'hydrodynamique est déterminée par un modèle de couche limite 2D à surface rigide, où sont résolues les équations de Reynolds ainsi que l'équation de conservation de la masse. L'effet de la déformation de la surface libre est négligeable pour des nombres de 


\section{XIII ${ }^{\text {èmes }}$ Journées Nationales Génie Côtier - Génie Civil \\ Dunkerque, 2-4 juillet 2014}

Froude inférieurs à l'unité (FREDSOE, 1982), ce qui est le cas pour les configurations étudiées $(0.2<F r<0.5)$. La turbulence est résolue par un modèle en $k-\omega$ mieux adapté que le modèle classique en $k-\epsilon$ pour les problèmes présentant des gradients de pression adverses comme cela est le cas au passage des crêtes des formes sableuses. Le modèle $k-\omega$ assure notamment une bonne description de la zone de recirculation (ANDERSEN, 1999). Le calcul de l'énergie cinétique turbulente $k$ et du taux de dissipation par la turbulence $\omega$ permet de déterminer la viscosité turbulente, $v_{t}=k / \omega$. Voir TJERRY (1995) et ANDERSEN (1999) pour plus de détail sur la résolution numérique du modèle.

\subsection{Module de transport sédimentaire}

Le transport par charriage est déterminé à l'aide de la formulation classique de MEYER-PETER \& MULLER (1948) adaptée aux nombres de Shields modérés. La concentration $c$ du sédiment est calculée via une équation d'advection-diffusion.

La concentration au fond $c_{b}$ étant calculée par le modèle d'ENGELUND \& FREDSØE (1976) à l'altitude $h+2 d_{50}$, où $h$ est l'altitude du fond et $d_{50}$ le diamètre médian du sédiment. Le flux de sédiment en suspension est ensuite calculé par intégration sur la verticale du flux horizontal de la concentration $c$.

\subsection{Module morphologique}

L'évolution du fond est déterminée à partir de l'équation de conservation du sédiment, résolue par un schéma centré non oscillant (NOCS) auquel est ajouté un module d'avalanche (MARIEU et al., 2008). Le schéma NOCS permet notamment de bien gérer les sauts de flux au niveau des crêtes des formes sableuses.

\subsection{Couplage}

Dans le cas des dunes hydrauliques, le modèle fonctionne de façon découplée entre les modules hydrodynamique et morphologique, la morphologie du fond étant mise à jour qu'à partir du moment où l'hydrodynamique a atteint un état stationnaire. Cette hypothèse est valable dans la mesure où les temps d'adaptation de l'hydrodynamique sont très petits devant les temps d'évolution du fond or les vitesses de migration des formes sableuses sont de l'ordre de $10^{-4} \mathrm{~m} / \mathrm{s}$ pour l'évolution présentée dans ce papier.

\section{Protocole expérimental}

Le modèle est utilisé pour reproduire des scénarios d'évolution du fond tirés d'expériences contrôlées en laboratoire. Les simulations ont pour but de vérifier l'aptitude du modèle à reproduire la physique du processus d'évolution d'un fond faiblement perturbé soumis à un courant stationnaire. Parmi les auteurs ayant étudié la physique des formes sableuses en laboratoire, peu traitent de façon détaillée le développement des dunes soit parce qu'ils n'atteignent pas la saturation (temps 


\section{Thème 2 - Dynamique sédimentaire}

d'évolution trop longs, carence du sédiment) soit parce que le protocole expérimental utilisé ne permet pas de suivre dans le détail les processus d'évolution des dunes. La simulation numérique permet l'étude de ces processus dans le détail et d'en comprendre les mécanismes.

\subsection{Paramètres de l'expérimentation:}

Le scénario modélisé s’inspire des expérimentations de COLEMAN et al. (2003), et plus particulièrement le cas R9. Les conditions expérimentales sont présentées dans le Tableau 1. Le transport en suspension est inclus. Les conditions expérimentales divergent des conditions de la simulation car le canal utilisé en laboratoire est fermé. Cela induit des profils de vitesse différents et des temps d'évolution plus rapides. COLEMAN et al. (2003) compare les évolutions respectives des dunes en canal ouvert et fermé pour des rapports de vitesses $u^{*} / u_{c}$ du même ordre de grandeur ( $u^{*}$ : vitesse de cisaillement et $u_{c}$ : vitesse critique de mise en mouvement) et montre des temps d'évolution plus rapides en canal fermé avec des formes sableuses de hauteur plus importante à l'équilibre (facteur 3 à 4) et de longueur d'onde un peu plus importante (facteur 1.5 à 2). Un autre facteur d'incertitude est le caractère légèrement tridimensionnel des dunes en laboratoire et des effets de bords non pris en compte dans les simulations.

Tableau 1. Conditions expérimentales.

\begin{tabular}{llllcc}
\hline Paramètre & $D(\mathrm{~m})$ & $U(\mathrm{~m} / \mathrm{s})$ & $d_{50}(\mathrm{~mm})$ & $u^{*} / u_{c}$ & $u^{*} / w_{s}$ \\
\hline Valeur & 0.1 & 0.297 & 0.11 & 1.27 & 1.4 \\
\hline
\end{tabular}

$D$ : profondeur ; $u_{*}:$ vitesse de cisaillement ; $d_{50}$ : diamètre médian du sédiment ; $u_{c}:$ vitesse critique de cisaillement ; $w_{s}$ : vitesse de chute du sédiment (loi de Stokes).

\subsection{Méthode de mesure:}

COLEMAN et al. (2003) utilise un protocole expérimental complexe (mesure du profil par visée laser et des vitesses par velocimètre doppler) lui permettant de suivre l'évolution du fond dès les premières rides d'instabilité jusqu'à la saturation du champ de dunes à intervalles de temps tels que l'écart de distance entre deux profils mesurés soit compris entre $1 \mathrm{~mm}$ et $2.5 \mathrm{~mm}$. Les formes sableuses sont identifiées par leurs fronts raides sur les profils mesurés et les altitudes des crêtes et des creux déterminés par les valeurs extrêmes entre deux formes sableuses (respectivement amont et aval). Les longueurs d'onde sont déterminées par analyse d'autocorrélation (COLEMAN \& MELVILLE, 1996). 


\section{XIII ${ }^{\text {èmes }}$ Journées Nationales Génie Côtier - Génie Civil \\ Dunkerque, 2-4 juillet 2014}

\section{Résultats et discussion}

\subsection{Observation}

L'expérimentation montre que les premières rides émergent du fond aléatoirement perturbé avec une longueur d'onde dépendant essentiellement du diamètre du sédiment (longueurs d'onde similaires en canal ouvert et fermé). Pour l'expérimentation R9, la longueur d'onde initiale équivaut à $\lambda=53 \mathrm{~mm}$. Ces premières rides développent un front d'avalanche de pente égale à l'angle de repos du sédiment et commencent à migrer. Les hauteurs hétérogènes conduisent à des vitesses de migration différentes et poussent les formes sableuses à interagir entre elles. Des appariements en cascade en résultent et entraînent la croissance des formes sableuses jusqu'à atteindre une longueur d'onde et une hauteur limite maintenues dans le temps $(\lambda \approx 0.14 \mathrm{~m}$ et une hauteur $H \approx 0.015-0.02 \mathrm{~m})$. Les structures obtenues ont une longueur d'onde comparable à la profondeur de la colonne d'eau et correspondent à un mode de dune (CHARRU, 2013).

\subsection{Résultats de la modélisation}

Les figures 1 à 3 présentent les résultats de la simulation. La figure 1 montre l'évolution temporelle du profil du fond. On retrouve les stades d'évolution décrits dans les expérimentations : d'abord (1) l'émergence rapide des premières rides développant un front d'avalanche, puis (2) la migration des formes de fond conduisant à des appariements en cascade (de $t \approx 100 \mathrm{~s}$ à $t \approx 1000 \mathrm{~s}$ ), jusqu'à obtenir (3) un champ de dunes interagissant entre elles sans s'apparier $(t>1000 \mathrm{~s})$. Dans cette dernière phase les formes sableuses semblent osciller autour d'une position d'équilibre correspondant à leur état à saturation. Les dunes les plus petites rattrapent les dunes situées en en aval de l'écoulement sans parvenir à les rejoindre et ces dernières accélèrent en diminuant de taille, pour croître à nouveau et atteindre l'état d'équilibre. La figure 3 présente l'évolution des caractéristiques physiques moyennes du fond en fonction du temps. Les 3 phases décrites précédemment y sont clairement identifiables. La phase initiale se caractérise par une croissance rapide de la hauteur moyenne et une longueur d'onde relativement stable (1), la hauteur et la longueur d'onde moyenne du champ de rides augmentent ensuite de manière significative pendant la phase d'appariements (2), avant de se stabiliser à saturation (3). La longueur d'onde des rides initiales $(\lambda \approx 55 \mathrm{~mm})$ est du même ordre de grandeur que celle mesurée lors des expérimentations ( $\lambda \approx 53 \mathrm{~mm})$. A l'équilibre, le champ de formes sableuses atteint une longueur d'onde moyenne $\lambda \approx 0.11 \mathrm{~m}$ et une hauteur $H \approx 0.005 \mathrm{~m}$. Les formes à saturation présentent un facteur d'échelle de 1.5 sur la longueur d'onde et 3 à 4 sur la hauteur par rapport au scénario R9, ce qui correspond aux différences évoquées au paragraphe 3.1 pour des expérimentations équivalentes en canal ouvert et fermé (COLEMAN et al., 2003). 


\section{Thème 2 - Dynamique sédimentaire}

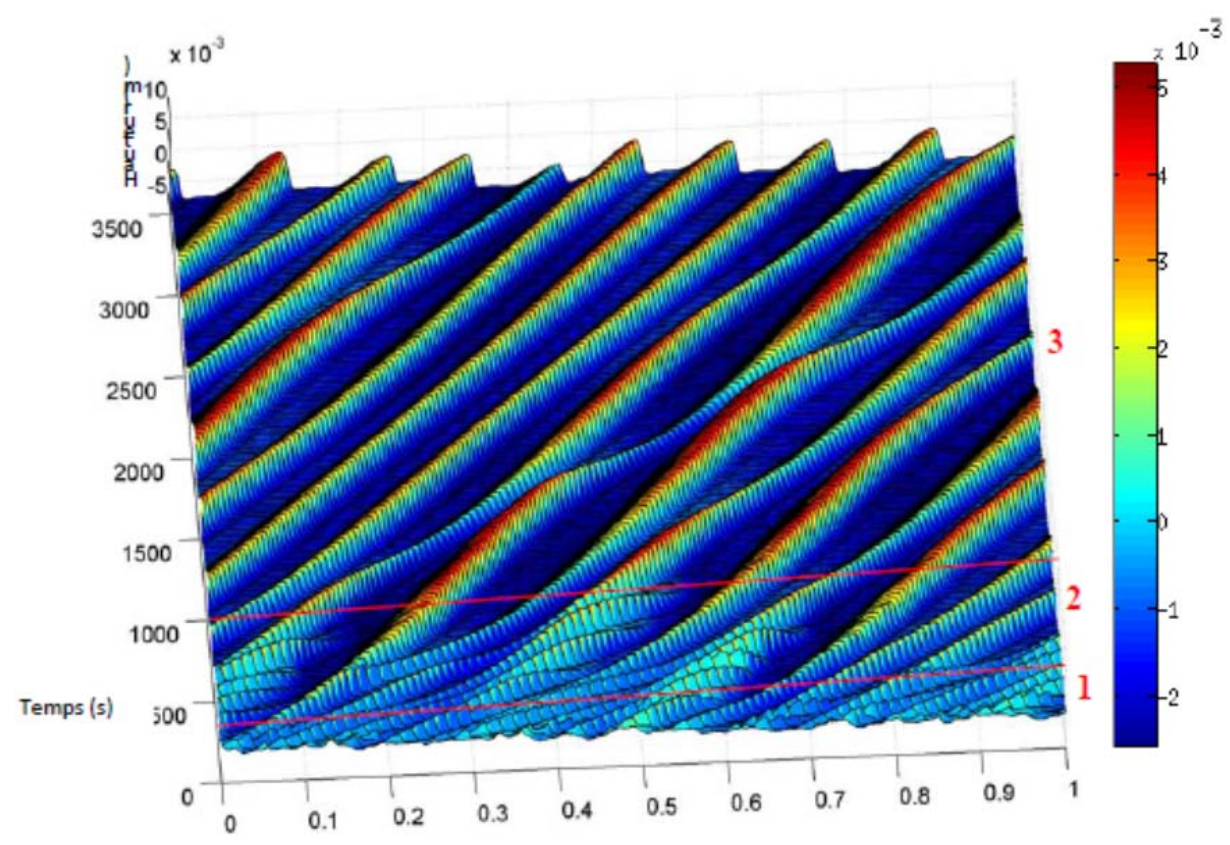

Figure 1. Evolution du champ de formes sableuses à partir du fond faiblement perturbé.
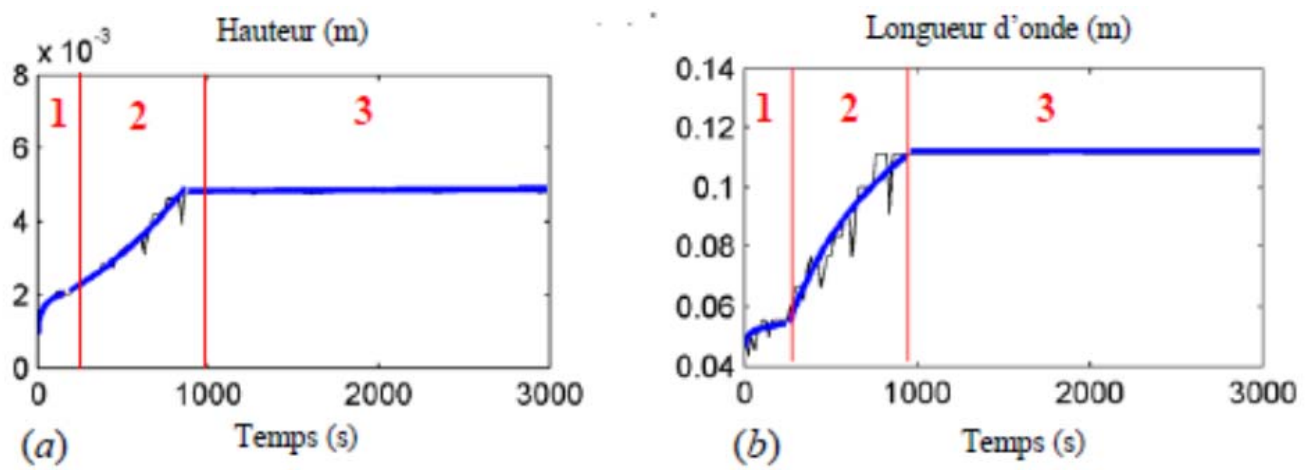

Figure 2. Evolution temporelle de (a) la moyenne des hauteurs du champ de dunes et (b) de leurs longueurs d'onde. Le trait noir montre le résultat brut et le trait bleu sa courbe de régression.

La figure 4 illustre une séquence d'appariement entre deux rides issue de la modélisation et permet de mieux comprendre sa phénoménologie. A $t=300 \mathrm{~s}$, la ride située en amont a une vitesse de migration supérieure à la ride en aval et la rattrape. Au fur et à mesure la bulle de recirculation de la ride en amont exerce un effet d'écran visà-vis de l'écoulement entraînant une diminution des vitesses le long du profil de la ride en aval et piégeant les sédiments. La célérité de la forme en aval diminue, sa crête se retrouve bientôt piégée dans la bulle de recirculation de la forme en amont et est érodée $(t=450 \mathrm{~s})$. La ride en aval s'aplatit et se désagrège laissant la ride en amont poursuivre sa migration pour bientôt laisser place à une seule forme sableuse. Cette séquence également décrite par BEST, (2013) est parfaitement reproduite par le modèle et montre de façon détaillée le mécanisme clé à l'origine de l'auto organisation du champ de dune. 


\section{XIII ${ }^{\text {èmes }}$ Journées Nationales Génie Côtier - Génie Civil \\ Dunkerque, 2-4 juillet 2014}

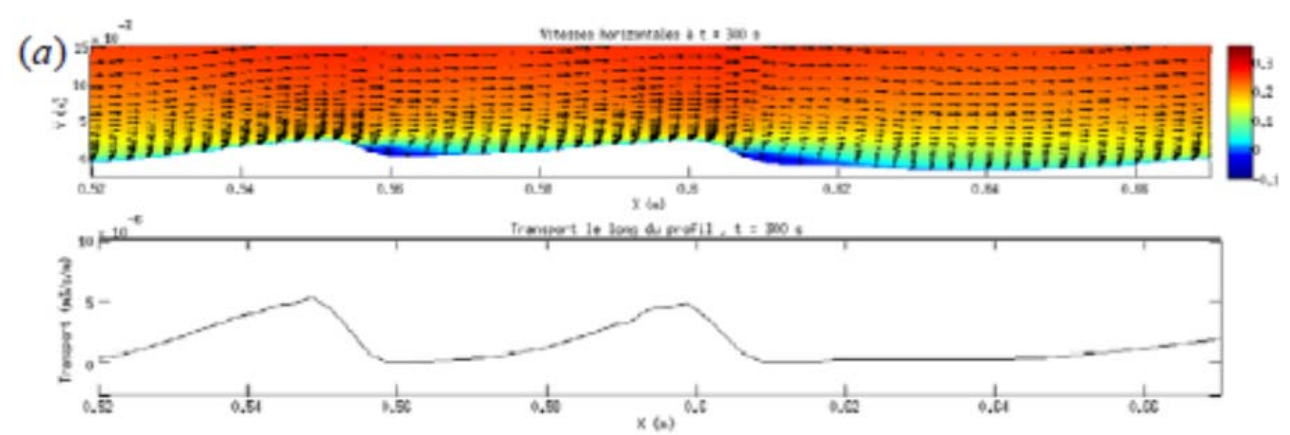

(b)
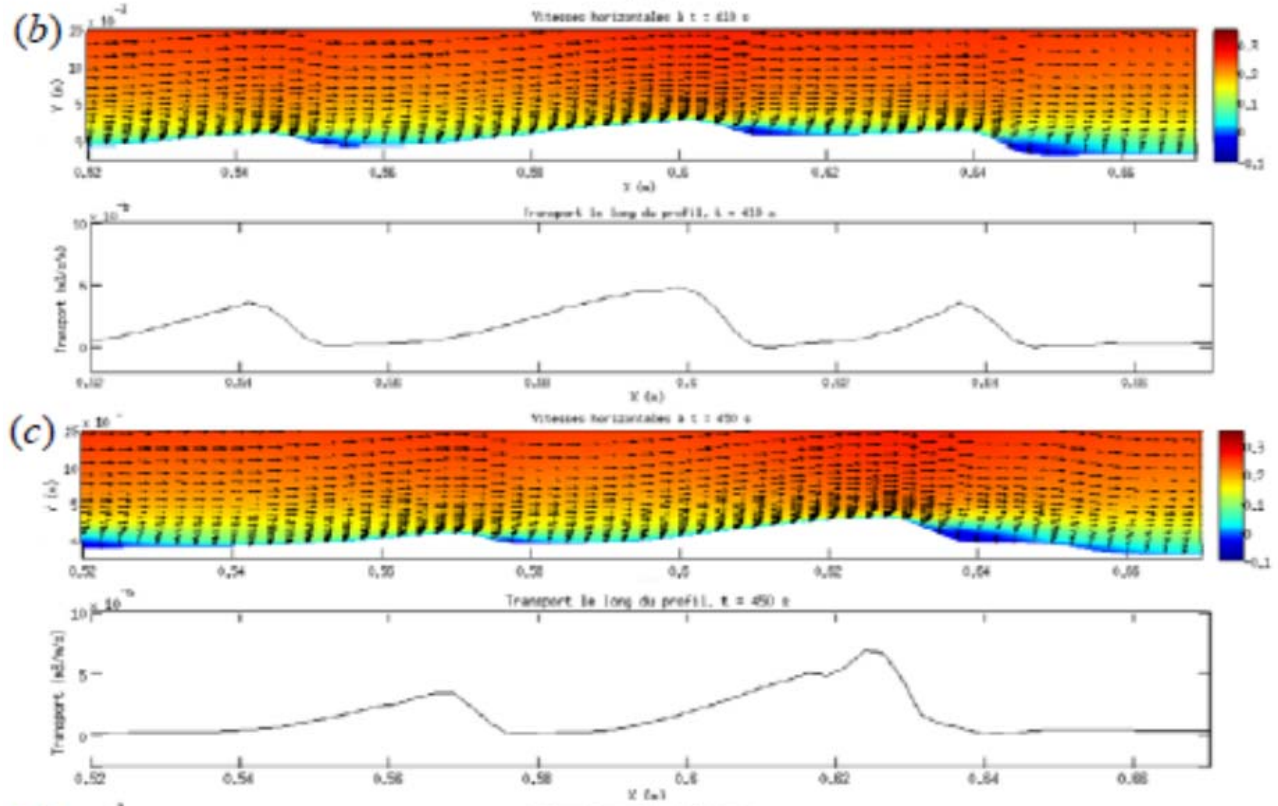

(d)
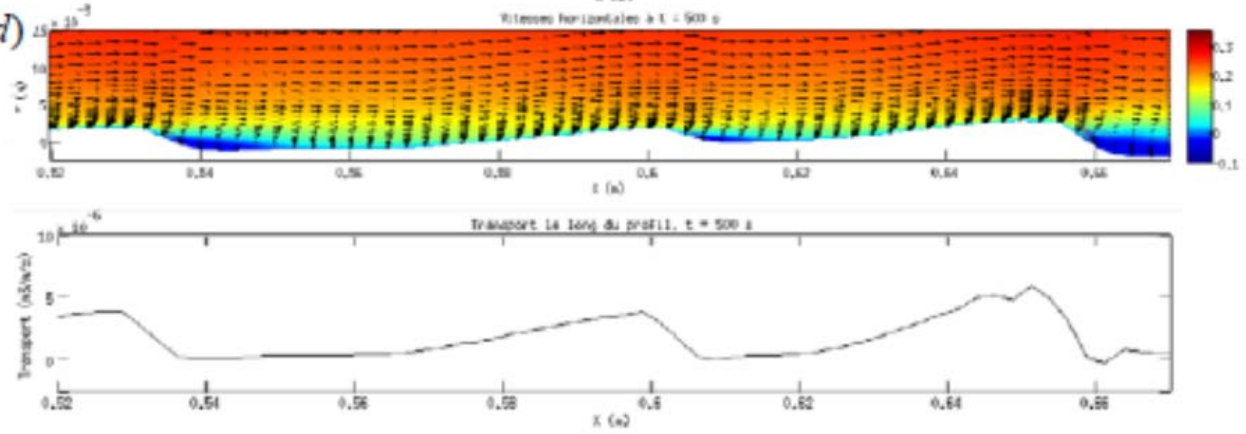

Figure 3. Champ des vitesses horizontales et transport total le long du profil de fond à ( a $t=300 \mathrm{~s}$, (b) $t=410 \mathrm{~s}$, (c) $t=450 \mathrm{~s}$ et (d) $t=500 \mathrm{~s}$.

\section{Conclusions}

En s'inspirant des paramètres d'une expérience en laboratoire, le modèle reproduit de façon satisfaisante l'évolution morphodynamique du fond faiblement perturbé jusqu'à l'équilibre. Les résultats de la simulation montrent les différentes phases de développement du fond, de l'apparition des premières rides jusqu'à la saturation du champ de dunes exhibant des caractéristiques physiques moyennes stationnaires. Le mécanisme d'appariement est reproduit et apparaît comme étant le processus clé 
permettant au champ de formes sableuses d'évoluer. Les résultats de la modélisation permettent d'expliquer en détail la phénoménologie derrière le processus d'appariement et notamment les interactions dynamiques à travers l'influence de la bulle de recirculation de la forme amont.

\section{Références bibliographiques}

ANDERSEN K.H. (1999). The dynamics of ripples beneath surface waves and topics in shell models of turbulence. Ph.D. dissertation, Det Naturvidenskabelige Fakultet Københavns Universitet, 1999.

BEST J. (2013). The dynamics of bedform amalgamation: new insights from a very thin flume. Proceedings of Marine and River Dune Dynamics,Bruges, Belgium, pp 29-34.

CHARRU F. (2013), Sand Ripples and Dunes, Annual Review of Fluid Mechanics, Vol. 45, pp 469-493. http://dx.doi.org/10.1146/annurev-fluid-011212-140806

COLEMAN S.E., MELVILLE B.W. (1996). Initiation of bedforms on a flat sand bed. Journal of Hydraulic Engineering, Vol. 122, pp 301-309. http://dx.doi.org/10.1061/(ASCE)07339429(1996)122:6(301)

COLEMAN S.E., FEDELE J.J., GARCIA M.H. (2003).Closed-Conduit Bed-Form Initiation and Development. Journal of Hydraulic Engineering, ASCE, Vol 129, pp 956964. http://dx.doi.org/10.1061/(ASCE)0733-9429(2003)129:12(956)

ENGELUND F. (1970). Instability of erodible beds. Journal of Fluid Mechanics, Vol 42, Part 2, pp 225-244. http://dx.doi.org/10.1017/S0022112070001210

FOURRIERE A. (2010). Bedforms in a turbulent stream : formation of ripples by primary linear instability and of dunes by nonlinear pattern coarsening. Journal of Fluid Mechanics, Vol. 649, pp 287-328. http://dx.doi.org/10.1017/S0022112009993466

FREDSOE J. (1982). Shape and dimensions of stationary dunes in rivers. Journal of hydraulic division. ASCE, Vol. 108, NO. HY8, 1982.

GIRI S., SHIMIZU Y. (2006). Numerical computation of sand dune migration with the free surface flow. Water resource research, Vol. 42(10), W10422. http://dx.doi.org/10.1029/2005WR004588

KENNEDY J.F. (1963). The mechanics of dunes and antidunes in erodible bed channels. Journal of fluid mechanics, Vol. 16, pp 521-544. http://dx.doi.org/10.1017/S0022112063000975

MARIEU V., BONNETON P., FOSTER D.L., ARDHUIN F. (2008). Modeling of vortex ripple morphodynamics. Journal of Geophysical Research,Vol. 113, C09007. http://dx.doi.org/10.1029/2007JC004659

NIEMANN S., FREDSOE J., JACOBSEN N.G. (2011). Sand Dunes in Steady Flow at Low Froude Numbers: Dune Height Evolution and Flow Resistance. Journal of Hydraulic Engineering, 137(1), pp 5-14. http://dx.doi.org/10.1061/(ASCE)HY.1943-7900.0000255

TJERRY S. (1995). Morphological calculation of dunes in alluvial rivers. Ph.D. dissertation, Technical University of Denmark, 1995. 